\title{
Efecto de la intervención nasal y nasosinusal en calidad de sueño en el Hospital Edgardo Rebagliati Martins 2017
}

\author{
Olenka Alcas* 1,a; Paul Rubén Alfaro-Fernández 2,b; Rosa Bertha Gutarra-Vilchez 2, b
}

RESUMEN

Objetivo: Determinar el efecto de la cirugía nasal y nasosinusal endoscópica sobre la calidad de sueño en el Servicio de Otorrinolaringología del Hospital Edgardo Rebagliati Martins durante el año 2017.

Materiales y métodos: Estudio retrospectivo y longitudinal. La muestra es de 92 pacientes operados en quienes se evaluó la calidad de sueño mediante el Índice de calidad de sueño de Pittsburgh. Los datos fueron analizados con SPSS versión 24 y se realizó análisis univariado, Mann-Whitney, correlación de Spearman y prueba de rango con signo de Wilcoxon. Resultados: La calidad de sueño mejoró después de la operación nasal y nasosinusal endoscópica $(p<0,01)$, al igual que la obstrucción nasal $(\mathrm{p}<0,01)$. En los datos preoperatorios, se encontró correlación entre IMC (índice de masa corporal) y obstrucción nasal con calidad de sueño.

Conclusiones: Las operaciones nasal y nasosinusal endoscópica tienen un efecto positivo sobre calidad de sueño en pacientes del Servicio de Otorrinolaringología del Hospital Edgardo Rebagliati Martins 2017.

Palabras clave: Sueño; Higiene del sueño; Cirugía endoscópica por orificios naturales (Fuente: DeCS BIREME).

\section{Effect of nasal and sinus surgery on sleep quality at the Hospital Edgardo Rebagliati Martins during 2017}

\section{ABSTRACT}

Objective: To determine the effect of nasal and sinus endoscopic surgery on sleep quality at the Otorhinolaryngology Department of the Hospital Edgardo Rebagliati Martins during 2017.

Materials and methods: A retrospective and longitudinal study was conducted with a sample of 92 patients who underwent surgery and whose sleep quality was assessed using the Pittsburgh Sleep Quality Index. The data was analyzed using software SPSS version 24. Additionally, a univariate analysis, Mann-Whitney U test, Spearman's rank correlation coefficient and Wilcoxon signed rank test were used in the study.

Results: Both sleep quality $(\mathrm{p}<0.01)$ and nasal obstruction $(\mathrm{p}<0.01)$ improved after nasal and sinus endoscopic surgery. The preoperative data shows that BMI (body mass index) and nasal obstruction have a correlation with sleep quality. Conclusions: Nasal and sinus endoscopic surgery had a positive effect on sleep quality in patients of the Otorhinolaryngology Department of the Hospital Edgardo Rebagliati Martins during 2017.

Keywords: Sleep; Sleep hygiene; Natural orifice endoscopic surgery (Source: MeSH NLM).

1. Hospital Edgardo Rebagliati Martins. Lima, Perú.

2. Universidad de San Martin de Porres. Lima, Perú.

a. Médico Otorrinolaringóloga.

b. Docente de Posgrado.

* Autor corresponsal 


\section{INTRODUCCIÓN}

La calidad de sueño es una evaluación subjetiva del dormir, cuya alteración puede llevar a consecuencias como fatiga diurna, disminución de energía, déficit de concentración y rendimiento laboral, depresión, obesidad y disminución de calidad de vida en general. Representa un fenómeno complejo, de difícil definición y medición. Incluye aspectos cuantitativos como duración, latencia o número de despertares y factores cualitativos como sensación de profundidad o descanso durante el sueño. La prevalencia de alteraciones en su calidad varía entre 15 y $35 \%$ (1), y puede estar influenciada por diferentes factores, uno de ellos es la resistencia nasal y patologías nasosinusales; existen estudios que reportan una mejoría después de intervenciones nasales ${ }^{(2)}$. Se ha descrito ampliamente en la literatura que pacientes con obstrucción nasal pueden presentar alteración de la calidad de sueño y de la vida en general. No obstante, aún son necesarios mayores estudios que identifiquen los mecanismos detrás de esta relación y mejorar el entendimiento del impacto relativo de la inflamación y obstrucción nasal sobre la vía aérea superior y sueño.

Se ha evaluado un impacto positivo de la septoplastia y la rinoplastia (1) en calidad de sueño, que pareciera estar relacionado al grado de obstrucción nasal previa. Otros estudios respecto a la intervención nasosinusal (operación endoscópica por sinusitis crónica), sugieren que hay un efecto positivo de calidad de sueño expresada por el paciente, pero que parece estar relacionada por el grado de severidad de sinusitis crónica, o ausencia o presencia de pólipos nasales ${ }^{(2-6)}$.

Existe controversia sobre el mecanismo en que estos procedimientos influyen en la calidad del sueño del paciente. No está claro si la mejoría es solo por la resolución de la obstrucción nasal, por el proceso de base (desviación de tabique nasal, presencia de pólipos), o por disminución de mediadores inflamatorios sistémicos, como ocurre después de una intervención endoscópica por sinusitis crónica ${ }^{(6)}$.

En nuestro país se da poca importancia a la calidad de sueño en la población y no se difunde información de los diversos desórdenes del sueño. Además, no hay ningún estudio sobre el efecto de una enfermedad o intervención nasal sobre el sueño. La operación nasal es una indicación frecuente en otorrinolaringología para pacientes que consultan por ronquido, somnolencia y alteración de sueño. Generalmente, "se asegura" al paciente que estos problemas serán solucionados después del procedimiento.

El objetivo del presente estudio es determinar el efecto de las intervenciones nasal y nasosinusal endoscópica sobre la calidad de sueño en el Servicio de Otorrinolaringología del
Hospital Edgardo Rebagliati Martins en 2017.

\section{MATERIALES Y MÉTODOS}

Diseño y población de estudio

Se realizó un estudio observacional, retrospectivo y longitudinal. La población estudiada fueron los pacientes asegurados del Servicio de Otorrinolaringología del Hospital Nacional Edgardo Rebagliati Martins, a quienes se les realizó septoplastia por tabique nasal desviado, operación nasosinusal endoscópica por sinusitis crónica con o sin poliposis, o una combinación de ambas, durante el año 2017.

El tamaño de muestra se calculó con nivel de confianza de $95 \%$, una precisión de 0,05 y 0,09 de efecto de mejora, de acuerdo a la literatura científica; además, con un tamaño muestral ajustado a pérdidas del $15 \%$, lo que da como resultado 92 casos.

La selección de la muestra se realizó por muestreo probabilístico simple a partir de todos los pacientes que fueron operados en el Servicio de Otorrinolaringología. Los criterios de inclusión fueron ser paciente mayor de 18 años, que ha tenido una septoplastía o intervención nasosinusal endoscópica y tener, por lo menos, un control postoperatorio con una diferencia de tres meses. Los criterios de exclusión fueron presentar una historia clínica y cuestionarios incompletos, diagnóstico de neoplasia maligna, algún desorden genético, proceso autoinmune o inmunodeficiencia como causa de sinusitis crónica, y haber tenido una operación nasal o nasosinusal secundaria.

\section{Variables y mediciones}

La calidad de sueño se midió con el índice de calidad de sueño de Pittsburgh ${ }^{(7,8)}$ y la obstrucción nasal con la escala de evaluación de los síntoma de obstrucción nasal (NOSE, por sus siglas en inglés) ${ }^{(9)}$. Ambas valoraciones fueron subjetivas.

El riesgo de tener apnea obstructiva del sueño fue medido mediante el cuestionario de Berlín ${ }^{(10,11)}$. La severidad de la sinusitis crónica se registró en la historia clínica mediante dos sistemas de clasificación ${ }^{(12)}$ : el endoscópico de LundKenedy y el tomográfico con la escala Lund-Mackay.

Los datos contenidos en la historia clínica y en los cuestionarios y escalas fueron registrados en una ficha diseñada para esta investigación. Se tomaron los valores preoperatorios y postoperatorios (de por lo menos tres meses de diferencia).

\section{Análisis estadístico}

Para el análisis se empleó la estadística descriptiva para las variables sexo, edad, peso, talla e IMC (índice de masa corporal). Además, se describieron las características basales 
preoperatorias de las variables obstrucción nasal, riesgo de apnea, severidad de sinusitis endoscópica y tomográfica, así como calidad de sueño. Se realizó la prueba de normalidad de Kolmogorov-Smirnov para las variables obstrucción nasal y calidad de sueño en el pre y postoperatorio. Se obtuvo un nivel de significancia menor a 0,05 con lo cual se rechaza la hipótesis nula, y se acepta que la muestra no sigue una distribución normal. Se utilizaron pruebas no paramétricas, la prueba de Mann-Whitney se usó para la comparación de medias de calidad de sueño entre pacientes con bajo y alto riesgo de apnea obstructiva de sueño. Además, se analizó la correlación entre IMC y obstrucción nasal con calidad de sueño preoperatoria y, entre los pacientes con diagnóstico de sinusitis crónica, se examinó la correlación entre severidad endoscópica y tomográfica con calidad de sueño preoperatoria, mediante el coeficiente de correlación de Spearman. Finalmente, se evaluó la diferencia de medias entre obstrucción nasal preoperatoria y postoperatoria, y calidad de sueño preoperatorio y postoperatorio mediante la prueba de rangos con signo de Wilcoxon.

\section{Consideraciones éticas}

Los nombres y apellidos de los participantes se cambiaron a códigos en las bases de datos para mantener la confidencialidad. Se obtuvo aprobación por el Comité de Capacitación e Investigación del Hospital Edgardo Rebagliati Martins.

\section{RESULTADOS}

Se evaluaron 92 pacientes con una edad media de $50,63+/$ 13,53 . El $56,5 \%$ (52) eran del sexo masculino y $60,9 \%$ (56) no tenía ningún antecedente patológico de importancia. Según el tipo de operación, el 41,3 \% (38) fueron sometidos a septoplastia. La media del peso fue $70,96+/-11,56$; la talla $1,62+/-0,07$; y el IMC, $26,67+/-3,42$.

Con respecto a las variables preoperatorias, tenemos que la media de la escala NOSE fue 73 , el índice de calidad de sueño fue 8 . El grupo con septoplastia e intervención endoscópica nasosinusal tenía peor calidad de sueño preoperatoria; mientras que el grupo operado de septoplastia tenía mayor obstrucción nasal preoperatoria (Tabla 1). Adicionalmente, se encontró que el $72,8 \%$ de todos los pacientes estudiados tenían bajo riesgo de tener apnea obstructiva del sueño.

Tabla 1. Características preoperatorias, según tipo de intervención, de pacientes con operación nasal y nasosinusal en Hospital Edgardo Rebagliati Martins, 2017

\begin{tabular}{|lcccc} 
& $\begin{array}{c}\text { Septoplastia } \\
\text { (Media, DE) }\end{array}$ & $\begin{array}{c}\text { Intervención } \\
\text { endoscópica } \\
\text { nasosinusal } \\
\text { (Media, DE) }\end{array}$ & $\begin{array}{c}\text { Septoplastia } \\
\text { (Media, DE) }\end{array}$ & $\begin{array}{c}\text { Total } \\
\text { (Media, DE) }\end{array}$ \\
\hline Calidad de sueño $†$ & $8,34 \pm 4$ & $6,9 \pm 3$ & $11,00 \pm 3$ & $8,35 \pm 4$ \\
\hline Obstrucción nasal * & $82,50 \pm 16$ & $65,88 \pm 22$ & $67,00 \pm 26$ & $72,99 \pm 22$ \\
\hline$x 2(2): 16.07, p<0,01 ;{ }^{*}{ }^{2} 2(2): 11,668, p<0,01$ & & &
\end{tabular}

De los pacientes con diagnóstico de sinusitis crónica sometidos a una operación endoscópica nasosinusal, se evidenció una media de severidad endoscópica de $6+/-3$, y una media de severidad tomográfica de $12+/-5$ (Tabla 2 ).

Tabla 2. Características endoscópicas y tomográficas en pacientes con operación endoscópica nasosinusal en Hospital Edgardo Rebagliati Martins -2017

\begin{tabular}{|c|c|c|c|}
\hline & $\begin{array}{c}\text { Operación endoscópica } \\
\text { nasosinusal } \\
\text { (Media, DE) }\end{array}$ & $\begin{array}{c}\text { Combinación } \\
\text { (Media, DE) }\end{array}$ & $\begin{array}{c}\text { Total } \\
\text { (Media, DE) }\end{array}$ \\
\hline Severidad endoscópica† & $7,18 \pm 3$ & $5,25 \pm 2$ & $6,46 \pm 3$ \\
\hline Severidad tomográfica* & $12,56 \pm 5$ & $9,80 \pm 4$ & $11,54 \pm 5$ \\
\hline †Z:-2,886, $p<0,01,{ }^{*} Z:-1,700, p>0,05$ & & & \\
\hline
\end{tabular}


La prueba de Mann-Whitney riesgo de apnea y calidad de sueño preoperatorio indicó que un bajo riesgo de apnea (rango promedio $=40,10$ ) tenía un menor índice de
Pittsburgh que aquellos pacientes con alto riesgo de apnea (Rango medio=63,64), $Z=-3,774, p<0,05$ (Tabla 3, Figura 1).

Tabla 3. Riesgo de apnea obstructiva del sueño y calidad de sueño preoperatoria en pacientes con operación endoscópica nasosinusal en Hospital Edgardo Rebagliati Martins -2017

\begin{tabular}{|lcccc|}
\hline & $\mathbf{n}$ & Media, DE & $\mathbf{Z}$ & p \\
\hline Bajo riesgo & 67 & $7,39 \pm 3,03$ & $-3,774$ & $<0,01$ \\
\hline Alto riesgo & 25 & $10,92 \pm 4,00$ & & \\
\hline
\end{tabular}

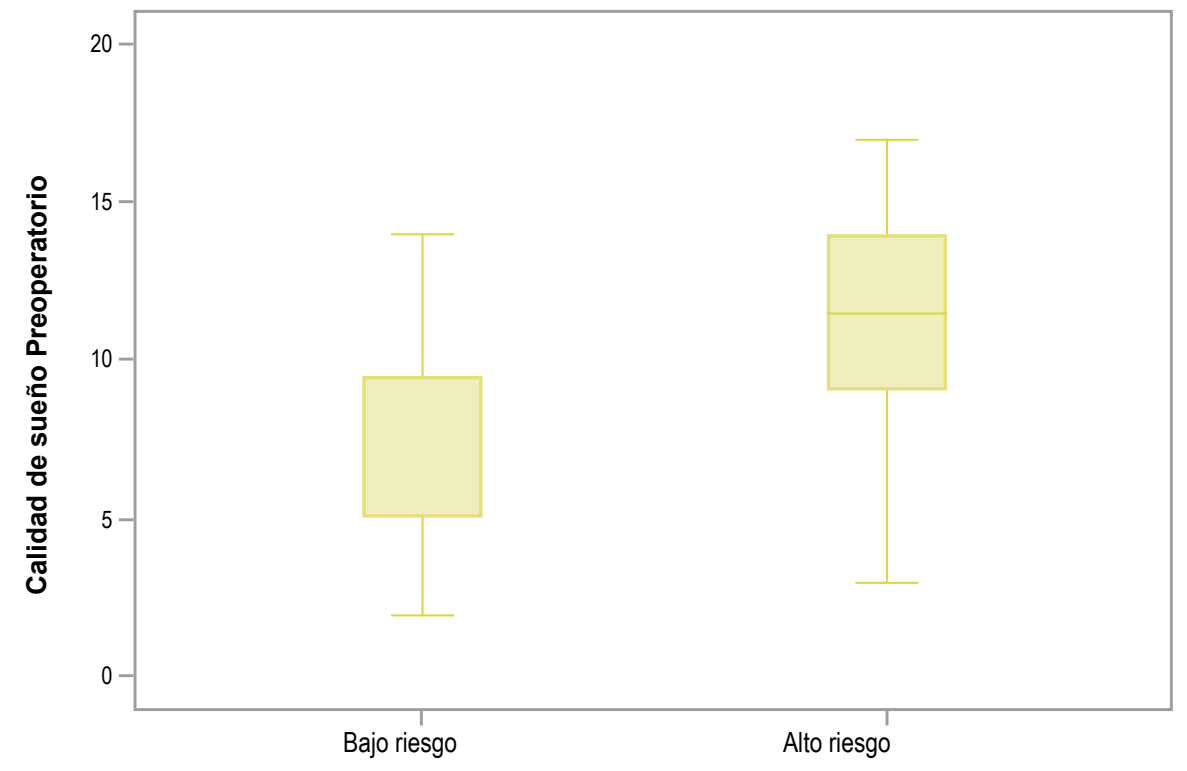

Riesgo de apnea obstructiva del sueño

Figura 1. Riesgo de apnea obstructiva del sueño y calidad de sueño preoperatorio de pacientes con operación nasal y nasosinusal en Hospital Edgardo Rebagliati Martins 2017

En el análisis de correlación de Spearman entre calidad de sueño preoperatoria e IMC se obtuvo un valor de 0,325 con $\mathrm{p}=0,002$, que indica una asociación media entre ambas variables. Entre calidad de sueño preoperatorio y obstrucción nasal, se obtuvo un valor de 0,453 con $p<0,05$, que implica una asociación positiva media entre ambas variables.

En el análisis de correlación de Spearman entre calidad de sueño preoperatorio y severidad endoscópica, se obtuvo un valor de $-0,143$ con $p=0,302$, es decir, no se encontró significancia estadística ni se evidenció correlación entre ambas variables. En el análisis entre calidad de sueño preoperatorio y severidad tomográfica, se obtuvo un valor de 0,154 con $p=0,266$, no se encontró significancia estadística. No se evidenció correlación entre ambas variables (Tabla 4). 
Tabla 4. Comparación de obstrucción nasal y calidad de sueño preoperatorio y postoperatorio de pacientes con intervenciones nasal y endoscópica nasosinusal en Hospital Edgardo Rebagliati Martins -2017

\begin{tabular}{|lcc} 
& $\begin{array}{c}\text { Calidad de sueño preoperatoria } \\
\text { Coeficiente de correlación }\end{array}$ & p valor \\
\hline IMC & 0,325 & 0,002 \\
\hline Obstrucción nasal & 0,453 & $<0,01$ \\
Severidad endoscópica & $-0,143$ & 0,302 \\
Severidad tomográfica & 0,154 & 0,266 \\
\hline
\end{tabular}

La prueba de rangos con signo de Wilcoxon indicó que la obstrucción nasal medida por la escala NOSE preoperatoria (rango medio $=46,50$ ) fue mayor que en el postoperatorio (rango medio $=0$ ), $Z:-8,336$ y $p<0,01 ;$ y que el índice de calidad de sueño preoperatorio (rango medio $=42,24$ ) fue mayor que en el postoperatorio (rango medio $=12,00$ ), $Z$ : $-7,784$ y $p<0,05$ (Tabla 5 ).

Tabla 5. Comparación de obstrucción nasal y calidad de sueño preoperatorio y postoperatorio de pacientes con operaciones nasal y endoscópica nasosinusal en Hospital Edgardo Rebagliati Martins -2017

\begin{tabular}{|lccc|} 
& $\begin{array}{l}\text { Preoperatorio }(\mathbf{n = 9 2}) \\
\text { Media, DE }\end{array}$ & Postoperatorio & P valor \\
\hline $\begin{array}{l}\text { Obstrucción nasal (Escala NOSE) } \\
\begin{array}{l}\text { Calidad de sueño (Índice de calidad } \\
\text { de sueño de Pittsburgh) }\end{array}\end{array}$ & $72,99 \pm 22,16$ & $21,28 \pm 10,98$ & $<0,01$ \\
\hline
\end{tabular}

\section{DISCUSIÓN}

Al usar el índice de calidad de sueño de Pittsburgh, se encontró un efecto positivo en los pacientes, después de las intervenciones nasal y nasosinusal endoscópica en el Servicio de Otorrinolaringología. Estos datos no solo reflejan una diferencia de medias significativa $(4,87$ postoperatorio, versus 8,35 preoperatorio), sino que también implica que, antes de la intervención, tenían mala calidad de sueño (valor menor a 6) que se corrigió después de la operación. Esta información es similar a la reportada en otros estudios (1-5,13-15). Los procedimientos corrigen el bloqueo nasal y mejoran la calidad de sueño en general.

Los participantes fueron, en la mayoría, varones, que corresponde a la población atendida por la seguridad social en el país. La edad media encontrada (50,63 años) fue mayor al promedio de la población titular asegurada (44 años) ${ }^{(16)}$. Un tercio de nuestros pacientes tenían alguna enfermedad previa, lo que se explica porque el Hospital Edgardo Rebagliati Martins es un centro de referencia nacional. La media de IMC se encontró en rango de sobrepeso, similar a lo reportado en población peruana ${ }^{(17)}$.

Respecto a las características preoperatorias, la muestra estudiada tuvo en promedio baja calidad de sueño medida por el índice de calidad de sueño de Pittsburgh, independientemente del tipo de operación. Estos datos coinciden con los diferentes estudios en pacientes sometidos tanto a intervenciones nasales (septoplastia, rinoseptolastia, reducción de cornetes) ${ }^{(1,18-20)}$ como a operación nasosinusal endoscópica por sinusitis crónica $(2,5,21)$.

También se reportó severidad en la obstrucción nasal mediante la escala NOSE $(\geq 30)$, un poco mayor en pacientes operados por septoplastia. Es importante señalar que uno de los principales síntomas en consulta es la obstrucción nasal, tanto en pacientes con sinusitis crónica como en aquellos con tabique nasal desviado, por lo que no es de sorprender que se haya encontrado valores altos en esta escala. La frecuencia de obstrucción 
nasal en centros urbanos es $26,7 \%{ }^{(22)}$, y alrededor de 75 a $80 \%$ de la población tiene algún grado de deformación nasal, principalmente, tabique desviado ${ }^{(23)}$. Las causas de la obstrucción son diversas, por ejemplo, congestión de la mucosa, tabique nasal desviado, hipertrofia turbinal, pólipos nasales, hipertrofia de adenoides y masas nasales. En la misma línea, la vía nasal es la ruta natural de la respiración, importante durante el sueño porque permite que la mandíbula cerrada mantenga fija la vía respiratoria superior. Si la vía nasal no está permeable, ocurre respiración oral que es inestable y altera la calidad de sueño ${ }^{(24)}$. En la muestra, encontramos solo dos causas: tabique nasal desviado y presencia de pólipos, que requirieron septoplastía y operación endoscópica nasosinusal, respectivamente.

Al considerar solo los pacientes con sinusitis crónica, los puntajes en severidad endoscópica (medida por LundKenedy) y severidad tomográfica (según Lund-Mackay), son similares a los encontrados en otras poblaciones $(5,25,26)$. Es importante señalar que los pacientes sometidos a una intervención son aquellos en los que falla el tratamiento médico, por eso los valores elevados.

Otra característica preoperatoria que se analizó fue el riesgo de apnea obstructiva del sueño. La mayoría de los participantes tenía bajo riesgo de apnea medido con el cuestionario de Berlín, pero al realizar comparación de medias se encontró que los pacientes con alto riesgo de apnea obstructiva del sueño tenían peor calidad de sueño preoperatoria. Esta característica puede causar confusión, ya que no se puede comprobar la existencia de apnea obstructiva del sueño con una prueba objetiva como la polisomnografía, por lo tanto, este dato se debe tomar con precaución.

Al respecto, un estudio evaluó el efecto de las intervenciones nasal y nasosinusal combinada en pacientes con diagnóstico de apnea obstructiva del sueño y encontró que hubo una mejoría leve de IAH (índice de apnea hipopnea), pero no curó el SAOS (síndrome de apnea obstructiva del sueño), ni tuvo un impacto clínico significativo ${ }^{(27,28)}$. En otro grupo de pacientes con SAOS, la intervención nasal mejoró, significativamente, los síntomas como ronquido, bloqueo nasal y síntomas relacionados al sueño ${ }^{(29)}$.

Un mayor valor en IMC estuvo asociado a una peor calidad de sueño, lo que concuerda con la literatura. Se ha evaluado que hay peor calidad de sueño con obesidad general y altos porcentajes de grasa corporal en adultos, después de controlar para variables sociodemográficas y estilos de vida. Esta asociación está dada principalmente por aumento en el tiempo de disfunción diurna, latencia de sueño y sus alteraciones ${ }^{(30)}$.

De igual forma, se halló una correlación significativa positiva entre obstrucción nasal y calidad de sueño preoperatoria. Un estudio encontró una asociación similar (31), y podría atribuirse a que un subdominio de la escala NOSE se correlaciona con las mediciones de Pittsburgh. Como ambos cuestionarios tienen ítems relacionados al sueño, es posible que se sobrepongan en algunas preguntas. En la escala NOSE, los ítems de congestión, obstrucción y problemas para respirar tienen alta correlación entre cada uno, pero muy poca con la pregunta relacionada al sueño. No se realizó el análisis individual de cada subítem a fin de establecer correlaciones más precisas.

Por otro lado, no se encontró correlación entre severidad endoscópica o tomográfica, y calidad de sueño, similar a lo reportado en otros estudios donde la severidad de la enfermedad medida por endoscopía, tomografía o función olfatoria no estuvo asociada a pobre calidad de sueño $(5,25)$. Solo un estudio reportó correlación significativa muy débil entre severidad endoscópica y síntomas relacionados al sueño, más no así a severidad tomográfica ${ }^{(26)}$. Estos hallazgos nos pueden hacer suponer que, en pacientes con sinusitis crónica, la obstrucción nasal influye en la calidad de sueño, pero sin correlación con la severidad de la enfermedad. La etiología y fisiopatología exacta se desconoce pero alguna teorías señalan que estaría en relación al grado de obstrucción nasal o en el caso de sinusitis crónica, por citoquinas inflamatorias que causan alteraciones en la fase de sueño REM, que en adultos está asociada a consolidación de la memoria ${ }^{(4)}$. Dentro del sistema inmunitario, los leucocitos producen citoquinas que tienen como función ser mediadores intercelulares y parecen jugar un rol en la regulación inmunitaria y del sueño ${ }^{(6)}$. Está demostrado que dentro de los estimulantes del sueño están la interleucina (IL), el interferón alfa y el factor de necrosis tumoral-a (TNF-a). Otras sustancias que estimulan el sueño (péptidos delta inductores del sueño, péptido muramil, colecistocinina, arginina vasopresina, péptido intestinal vasoactivo, hormona liberadora de la hormona de crecimiento, prostanglandina $D$, óxido nítrico y adenosina) están aumentadas durante la vigilia prolongada o en el curso de infecciones ${ }^{(32)}$.

En relación con el efecto de las intervenciones nasal y nasosinusal en obstrucción nasal, se encontró una mejoría significativa. Este hallazgo está relacionado con la mejoría de la calidad de sueño postoperatoria. Esto podría sugerir que aquellos con obstrucción nasal pueden experimentar un beneficio secundario en calidad de sueño luego de un procedimiento quirúrgico nasal o nasosinusal. Muchos de los pacientes pueden no estar conscientes de esta asociación, y no dar información sobre su calidad de sueño a menos que se les pregunte. Debido a los problemas asociados, los pacientes con obstrucción nasal deberían ser preguntados sobre alteraciones del sueño; así como pacientes con problemas en el dormir deben tener un examen exhaustivo de la vía aérea nasal. 
Las fortalezas del estudio incluyen un número de muestra representativa, recolección de datos solo por médicos especialistas en otorrinolaringología, y la utilización de instrumentos validados para obstrucción nasal, riesgo de apnea obstructiva del sueño y calidad de sueño. No obstante, existen limitaciones para la interpretación de los hallazgos. Primero, al ser un estudio retrospectivo, no se puede determinar relación causa-efecto entre los factores evaluados. Segundo, los hallazgos están circunscritos a una población que es operada en el Servicio de Otorrinolaringología de un hospital de referencia, por lo cual muchos de los pacientes ya tendrían mayor grado de obstrucción nasal y calidad de sueño que el de la población similar con el mismo problema. Se debe de tener cautela al extrapolar estos resultados a todos los pacientes con operaciones nasal o nasosinusal endoscópica. Finalmente, la medición de calidad de sueño fue subjetiva por el índice de calidad de sueño de Pittsburgh. La medición objetiva de calidad de sueño se debería realizar mediante la polisomnografía, por lo cual no sabemos si los pacientes con mala calidad de sueño tenían o no apnea obstructiva del sueño.

En conclusión, las intervenciones nasal y nasosinusal endoscópica tiene un efecto positivo sobre calidad de sueño en pacientes del Servicio de Otorrinolaringología del Hospital Edgardo Rebagliati Martins. Los pacientes con baja calidad de sueño tienen mayor riesgo de apnea obstructiva. Existe una asociación significativa entre IMC y calidad de sueño preoperatorio, y entre obstrucción nasal y calidad de sueño preoperatorio, pero no existe asociación entre severidad endoscópica y tomográfica, y calidad de sueño preoperatoria para la muestra estudiada.

Con base en los hallazgos, se debería preguntar sobre alteraciones del sueño en pacientes que acuden a consulta por obstrucción nasal, y futuros estudios deberían incluir mediciones objetivas de calidad de sueño mediante polisomnografía.

\section{REFERENCIAS BIBLIOGRÁFICAS}

1. Stapleton AL, Chang YF, Soose RJ, Gillman GS. The impact of nasal surgery on sleep quality: a prospective outcomes study. Otolaryngol Head Neck Surg. 2014; 151(5): 868-73.

2. Tosun F, Kemikli K, Yetkin S, Ozgen F, Durmaz A, Gerek M. Impact of endoscopic sinus surgery on sleep quality in patients with chronic nasal obstruction due to nasal polyposis. J Craniofac Surg. 2009; 20(2): 446-9.

3. Uz U, Günhan K, Yılmaz H, Ünlü H. The evaluation of pattern and quality of sleep in patients with chronic rhinosinusitis with nasal polyps. Auris Nasus Larynx. 2017; 44(6): 708-12.

4. Rotenberg BW, Pang KP. The impact of sinus surgery on sleep outcomes. Int Forum Allergy Rhinol. 2015; 5(4): 329-32.

5. Alt JA, Smith TL, Mace JC, Soler ZM. Sleep quality and disease severity in patients with chronic rhinosinusitis. Laryngoscope. 2013; 123(10): 2364-70.
6. Orb Q, Orlandi RR, Alt JA. Sleep dysfunction and its association to chronic rhinosinusitis: updated review. Laryngoscope Investig Otolaryngol. 2017; 2(2): 46-52.

7. Buysse DJ, Reynolds CF, Monk TH, Berman SR, Kupfer DJ. The Pittsburgh Sleep Quality Index: a new instrument for psychiatric practice and research. Psychiatry Res. 1989; 28(2): 193-213.

8. Escobar-Córdoba F, Eslava-Schmalbach J. Validación colombiana del índice de calidad de sueño de Pittsburgh. Rev Neurol. 2005; 40(3): 150-5.

9. Larrosa F, Roura J, Dura MJ, Guirao M, Alberti A, Alobid I. Adaptation and validation of the Spanish version of the Nasal Obstruction Symptom Evaluation (NOSE) scale. Rhinology. 2015; 53(2): 176-80.

10. Polanía-Dussan IG, Escobar-Córdoba F, Eslava-Schmalbach J, Netzer NC. Colombian validation of the Berlin questionnaire. Rev Fac Med. 2013; 61(3): 231-8.

11. Friedman $M$, Wilson $M N$, Pulver T, Pandya $H$, Joseph NJ, Lin $\mathrm{HC}$, et al. Screening for obstructive sleep apnea/hypopnea syndrome: subjective and objective factors. Otolaryngol Head Neck Surg. 2010; 142(4): 531-5.

12. Orlandi RR, Kingdom TT, Hwang PH, Smith TL, Alt JA, Baroody FM, et al. International Consensus Statement on Allergy and Rhinology: Rhinosinusitis. Int Forum Allergy Rhinol. 2016; 6(Suppl.1): S22-209.

13. Benninger MS, Khalid AN, Benninger RM, Smith TL. Surgery for chronic rhinosinusitis may improve sleep and sexual function. Laryngoscope. 2010; 120(8): 1696-700.

14. Nguyen D, Arous F, Gallet P, Felix-Ravelo M, Nguyen-Thi PL, Rumeau $C$, et al. Sinonasal symptom-related sleep disorders before and after surgery for nasal polyposis. Rhinology. 2017; 55(3): 262-8.

15. Bugten V, Nilsen AH, Thorstensen WM, Strand Moxness MH, Amundsen MF, Nordgård S. Quality of life and symptoms before and after nasal septoplasty compared with healthy individuals. BMC Ear Nose Throat Disord. 2016; 16: 13.

16. EsSalud. Características demográficas, sociales, económicas y acceso a los servicios de salud de la población asegurada a EsSalud [Internet]. Lima; 2015. Disponible en: http://www. essalud.gob.pe/downloads/estadistica/caract_demog_soc_ econ_acc_servic_salud_poblac_asegurada_essalud.pdf

17. World Health Organization. Peru [Internet]. World Health Organization; 2015. Disponible en: http://www.who.int/ countries/per/en/

18. Schwentner I, Dejakum K, Schmutzhard J, Deibl M, Sprinzl GM. Does nasal septal surgery improve quality of life?. Acta Otolaryngol. 2006; 126(7): 752-7.

19. Kara M, Erdoğan H, Güçlü O, Sahin H, Dereköy FS. Evaluation of sleep quality in patients with nasal septal deviation via the Pittsburgh Sleep Quality Index. J Craniofac Surg. 2016; 27(7): 1738-40.

20. Lindsay RW. Disease-specific quality of life outcomes in functional rhinoplasty. Laryngoscope. 2012; 122(7): 1480-8.

21. Sukato DC, Abramowitz JM, Boruk M, Goldstein NA, Rosenfeld RM. Endoscopic sinus surgery improves sleep quality in chronic rhinosinusitis: a systematic review and meta-analysis. Otolaryngol Neck Surg. 2018; 158(2): 249-56.

22. Jessen M, Malm L. Definition, prevalence and development of nasal obstruction. Allergy. 1997; 52(Suppl 40): 3-6.

23. Dinis PB, Haider H. Septoplasty: long-term evaluation of 
results. Am J Otolaryngol. 2002; 23(2): 85-90.

24. Gulec TC, Yoruk O, Gulec M, Selvi Y, Boysan M, Oral E, et al. Benefits of submucous resection on sleep quality, daytime and dream anxiety in patients with nasal septal deviation. Sleep Biol Rhythms. 2013; 11(4): 227-36.

25. Deepthi NV, Menon UK, Menon IR. Correlations and comparison between repeat computed tomography scores, endoscopy scores and symptomatic improvement before and after endoscopic sinus surgery: a pilot study. Clin Rhinol. 2013; 6(1): 32-40.

26. El Rassi E, Mace JC, Steele TO, Alt JA, Smith TL. Improvements in sleep-related symptoms after endoscopic sinus surgery in patients with chronic rhinosinusitis. Int Forum Allergy Rhinol. 2016; 6(4): 414-22.

27. Yalamanchali S, Cipta S, Waxman J, Pott T, Joseph N, Friedman $M$. Effects of endoscopic sinus surgery and nasal surgery in patients with obstructive sleep apnea. Otolaryngol Head Neck Surg. 2014; 151(1): 171-5.

28. Moxness MH, Nordgård S. An observational cohort study of the effects of septoplasty with or without inferior turbinate reduction in patients with obstructive sleep apnea. BMC Ear Nose Throat Disord. 2014; 14: 11.

29. Wu J, Zang HR, Wang T, Zhou B, Ye JY, Li YC, et al. Evaluation of the subjective efficacy of nasal surgery. J Laryngol Otol. 2017; 131(1): 37-43.

30. Rahe C, Czira ME, Teismann H, Berger K. Associations between poor sleep quality and different measures of obesity. Sleep Med. 2015; 16(10): 1225-8.

31. Thomas AJ, Orlandi RR, Ashby S, Mace JC, Smith TL, Alt JA. Nasal obstruction has a limited impact on sleep quality and quality of life in patients with chronic rhinosinusitis.
Laryngoscope. 2016; 126(9): 1971-6.

32. Sociedad Española del Sueño. Tratado de Medicina del sueño. Madrid: Editorial Médica Panamericana; 2015. 1196 p.

Fuentes de financiamiento:

Este artículo ha sido financiado por los autores.

Conflictos de interés:

Los autores declaran no tener conflictos de interés.

\section{Correspondencia:}

Olenka Alcas

Dirección: Jr. Huiracocha 2335 Jesús María. Lima, Perú.

Celular: +51997884437

Correo: olenka_14@hotmail.com

Recibido: 28 de marzo de 2019. Evaluado: 15 de junio de 2019. Aprobado: 14 de agosto de 2019.

(c) La revista. Publicado por Universidad de San Martín de Porres, Perú. (c) ${ }_{\text {Br }}$ Licencia de Creative Commons Artículo en acceso abierto bajo términos de Licencia Creative Commons Atribución 4.0 Internacional. (http://creativecommons.org/licenses/by/4.0/)

\section{ORCID iDs}

Olenka Alcas

Paul Rubén Alfaro-Fernández

Rosa Bertha Gutarra-Vilchez

https://orcid.org/0000-0003-2336-9495 https://orcid.org/0000-0002-8745-8270 https://orcid.org/0000-0002-5828-8380 